\title{
The Importance of a Multidisciplinary Clinical Evaluation in Palliative Care: Two Clinical Cases with Bone Fracture
}

\author{
Fonseca $\mathbf{V}^{1}$, Durão $\mathrm{S}^{1}$, Moura $\mathrm{I}^{1}$, Sidiropoulou $\mathbf{Z}^{1}$, Martins $\mathrm{A}^{1}$, Caetano $\mathrm{A}^{2}$ and Guimarães \\ Consciência ${ }^{2}$ \\ ${ }_{1}^{1}$ Department of Oncology, Centro Hospitalar Lisboa Ocidental, Estrada do Alto do Duque Fort, 1449-005 Lisbon, Portugal \\ ${ }^{2}$ Department of Orthopedics, Centro Hospitalar Lisboa Ocidental, Estrada do Alto do Duque Fort, 1449-005 Lisbon, Portugal
}

${ }^{*}$ Corresponding author: Fonseca V, Department of Oncology, Centro Hospitalar Lisboa Ocidental, Estrada do Alto do Duque Fort, 1449-005 Lisbon, Portugal, Tel: 210431000; E-mail: medicinavf@yahoo.com

Received: September 15, 2017; Accepted: September 29, 2017; Published: October 05, 2017

Citation: Fonseca V, Durão S, Moura I, Sidiropoulou Z, Martins A, et al. (2017) The Importance of a Multidisciplinary Clinical Evaluation in Palliative Care: Two Clinical Cases with Bone Fracture. Ann Clin Lab Res Vol.5:No.3:197.

\section{Abstract}

Background: Palliative and oncology units should work in a multidisciplinary organization, this being the best clinical practice actually known. Multidisciplinary osteo oncology units specialized in oncologic and palliative care with the capacity for taking critical decisions is really something new in our clinical practice. Nevertheless, the oncologic patient's approach, under palliative strategy and with a bone fracture is not always straightforward, as it requires complex decision-making that demand a multidisciplinary engagement.

Case study: We present different clinical cases where we explain how we are working in taking critical decisions in palliative care breast patients with bone fracture. In the last decades, in Portugal, there has been a change of the paradigm, in which medical oncology is concerned. It has been taking over a role each time more predominant in the clinical dynamics, serving as a connecting link among the different fields. The integration of palliative care in medical oncology practice, contributes to an improvement of the patients' quality of life and survival. Nonetheless, it should be based upon the medical evidence. The critical decision in palliative care imposes a profound reflection as it has a real impact, which cannot be underestimated. Therefore, it is important its discussion within the team, taking into consideration the patient's global vision and the real knowledge of the technical capacities in the hospital where the patient sought treatment. We still consider that the maintenance of the patient's contact with the hospital, physicians and medical oncology assistants, assumes a fundamental character, which reinforces its importance in the palliative care sector.

Conclusion: The different therapeutic options provided for the patients want to fulfill the purpose of improving their quality of life and survival. However, they only became achievable through the multidisciplinary evaluation carried out in a clinical context, which forces us to emphasize its crucial character, not only in the clinical approach of the oncological patients as well as those who are selected for palliative care.

Keywords: Multidisciplinary clinical evaluation; Palliative care; Clinical cases; Bone fracture

\section{Introduction}

Palliative and oncology units should be working together in a multidisciplinary organization, this being the best clinical practice currently known [1-3]. Multidisciplinary osteo oncology units specialized in oncological and palliative care capable of making critical decisions are a relatively new element of our daily clinical practice $[4,5]$.

For the majority of patients in palliative care a "less is more" approach is most effective (for example the potential disadvantages of polymedication) however, in particular cases, a more complex and/or invasive solution may be required. The advantage of a multidisciplinary clinical team is that it can use the individual experience and skills of its members to ascertain the positive and negative impacts of proposed treatments in order to decide upon a personalized approach for each patient. In clinical decision meetings, the oncologist presents the clinical case, emphasizing performance status and natural history of disease, whilst other healthcare professionals, such as social assistants and radiologists, contribute by providing information concerning the patient's home environment and medical images respectively $[6,7]$.

In this way, this publication intends to emphasize the relevance of integrating palliative care training into the oncology sector, especially among professionals who, upon becoming part of treatment teams, need to make critical decisions on a daily basis $[3,8]$.

In order to demonstrate this, this study presents two cases of patients suffering from breast cancer, a disease which is associated with a higher risk of pathological and/or iatrogenic fractures following osteopenia, a problem often caused by 
hormonal therapy [4-7]. Through these case studies, this publication explains how, in situations where our palliative and oncological patients suffer from bone disease, we proceed as far as clinical decision making, and highlights the benefits of a multidisciplinary clinical team in this process.

\section{Clinical Case 1}

A 71-year-old female patient with a right breast tumor diagnosed in 1998 (G2, hormone receptor positive and with sub expression CERB2 negative), underwent a modified radical mastectomy, CT chemotherapy, adjuvant RT radiotherapy and HT hormone therapy.

After a dysphonia episode in 2012 a mediastinum ganglionic relapse was discovered and metastatic disease was confirmed through biopsy. In line with this result, the patient's treatment options were several CT, RT and HT lines, which succeeded in obtaining the disease's analytical and imagiological clinical stability until 2015.

Meanwhile, in February 2014, following the appearance of focal deficit, the patient was evaluated with a Head CT scan which revealed 3 space-occupying lesions, highly suggestive of brain metastasis. In the absence of surgical indication a holocranial RT with palliative intent was then performed. Despite this, there was a progressive deterioration in the patient's general clinical condition and, without a significant improvement of the mentioned focal deficit, she was referred to palliative care with the proposal of a symptomatic/palliative approach.

Here it is important to note that the patient kept her normal follow-up with the medical oncology team at the clinical centre where she was integrated, despite also joining a continuing/ palliative care network.

In July 2015, she suffered a traumatic fall and sustained a right femoral neck fracture which, under normal circumstances, would be treated with surgery. Considering the patient's clinical context, she was evaluated by a multidisciplinary team and assigned for a cemented hip hemiarthroplasty which was carried out with no complications.

Five months after surgery, the patient was able to mobilize herself using a wheelchair, with no complaints of pain. She was also able to maintain an adequate social life at the institution where she was integrated to the obvious satisfaction of both herself and her relatives.

\section{Clinical Case 2}

A 34-year-old female patient, diagnosed with left breast carcinoma (G3, hormone receptor negative and with sub expression CERB2 positive) in 2013, underwent breast conserving surgery in another institution and was then redirected to our hospital.

In May 2013 she was submitted to a skeletal full body scintigraphy as well as an MRI which revealed multifocal bone dissemination. A discrete fracture at T8's inferior somatic platform (Type A: AO classification), was also noticed, with no evidence of medullar compression.

The clinical situation was evaluated by the multidisciplinary team, which considered it unnecessary to refer the patient for an orthopedic surgical procedure.

After performing 6 cycles of 21/21 days with QT FEC regime (Fluorouracil $(500 \mathrm{mg} / \mathrm{m} 2)$, Epirubicin (100 mg/m2), Cyclophosphamide $(500 \mathrm{mg} / \mathrm{m} 2)$ ] and having initiated the monoclonal antibody Denosumab (RANKL Inhibitor), the patient was referred for treatment with palliative RT centered at the vertebral fracture in October 2013.

Subsequently, she still completed biological therapeutics with monoclonal antibodies (Trastuzumab) and several CT regimes (weekly paclitaxel and oral capecitabine), with a confirmed regression of bone lesion and systemic disease control.

During this therapy, the patient was able to maintain her social life, even being able to enjoy several trips with her relatives.

Finally, in April 2015 multiple brain metastases occurred and despite the advised holo-cranial RT and radiosurgery, the patient passed away in September 2015, due to the progression of the aforementioned brain disease.

\section{Discussion}

As far as medical oncology is concerned, there has been a significant evolution in Portugal in the last few decades. We have seen it taking on a more and more predominant role in clinical dynamics and serving as a link between different fields.

The integration of palliative care in medical oncology practice contributes to an improvement in patients' quality of life as well as overall survival. Nonetheless, it must be based upon medical evidence [1].

Complex clinical situations in palliative care require the full involvement of multidisciplinary decision making, which becomes a fundamental step in the integrated clinical palliative care process [9].

In these contexts, the oncologist with a sub-specialization in palliative care becomes the corner stone of this multidisciplinary team. However, in our practice, when dealing with patients with bone disease, our multidisciplinary team includes not only medical oncologists, orthopedic surgeons and palliative care specialists, but also a large range of professionals from radiation oncologists and radiologists to physiatrist/physiotherapists, social assistants and psychiatrist/ psychologists. This so-called global approach is applied to our patients whether they are being treated within the hospital facilities or in an outpatient unit linked to the hospital, in this way we provide them with the best possible support care.

The first clinical case raises the problem of surgical indication for a femoral neck fracture in a patient under palliative care for breast cancer and brain metastasis. 
We admit that this fracture's etiology was partly related to the administration of $\mathrm{HT}$ as an adjuvant in a metastatic context. When treated with holo-cranial radiotherapy the expected survival of a breast cancer patient with brain metastasis is around 4 to 6 months after diagnosis of metastasis, which in this case took place approximately 3 months before [10].

Despite the expected survival and adopted palliative strategy, upon multidisciplinary evaluation we considered that an orthopedic surgery would probably grant her better pain control and consequently a better quality of life $[11,12]$.

Five months after the surgery she was integrated in an appropriate residence, and was undergoing a physiotherapy program, describing effective pain control and keeping an acceptable social life with relatives as well as healthcare professionals.

With regards to this first case then, the authors intend to raise the following question: by submitting a patient with a few months expected survival and in palliative strategy to a major surgery, are we really upholding her quality of life?

Critical decision making in palliative care requires profound reflection which considers its real impact and cannot be underestimated. This heightens the importance of a multidisciplinary discussion, which takes patient's global evaluation into consideration as well as real knowledge of the technical capacities at the Hospital where the patient sought treatment [13].

In this case, coming up with such a personalized and proactive clinical strategy would only be possible through the complementary actions of professionals from several medical fields of knowledge within the same hospital, who all have the same final objective in view.

We concluded that a palliative critical approach ended up being positive for the patient, as far as quality of life was concerned (better pain control, less observations at the emergency department and better social life).

Consequently, we still consider that maintaining contact with the hospital, physicians and medical oncology assistants is becoming crucial and increasingly relevant in palliative care.

Yet in the second case, which regards a patient who presented metastatic bone fracture breast carcinoma, it was decided not to perform a surgical treatment and administer radiotherapy as well as systemic therapeutics as palliative options. This highlights the idea that often for patients in palliative care, a "less is more" approach is more appropriate, as more invasive procedures were not deemed to provide any further benefits for the patient.

Metastatic breast cancer, presenting a survival rate of 2 to 3 years, implies a non-curative strategy with palliative alternatives that should be related to the tumor's biology and the extent of the disease. However, the purpose of therapy is prolonged survival and improved quality of life.

These patients are indicated for maximum medical investment whenever the required clinical condition is present, making it viable. In our clinical practice, these patients are usually referred to Palliative Care units especially when they are not indicated for specific oncological treatment.

It seems obvious that in this case the patient benefited from the treatment provided, presenting a better control of the disease's bone involvement and maintaining a good quality of life as desired.

\section{Conclusion}

The different therapeutic options provided for both patients have fulfilled the purpose of improving their quality of life as well as overall survival. However, this could only be obtained through a multidisciplinary evaluation carried out in an adequate clinical context. This of course reinforces its crucial value in selecting treatment for oncological patients, including those under Palliative Care. Furthermore, it suggests that maintaining a link between these patients and a Multidisciplinary Clinical Unit is a crucial point in therapeutic process, and most certainly a worthwhile asset for investment considering a future investigation hypothesis.

\section{References}

1. Vanbutsele G, Van Belle S, De Laat M, Surmont V, Geboes K, et al. (2015) The systematic early integration of palliative care into multidisciplinary oncology care in the hospital setting (IPAC), a randomized controlled trial: the study protocol. BMC Health Serv Res. 15: 554

2. Ibrahim T, Flamini E, Fabbri L, Serra P, Mercatali L, et al. (2009) Multidisciplinary approach to the treatment of bone metastases: Osteo-oncology center, a new organizational model. Tumori 95: 291-297.

3. National Institute for Health and Care Excellence (2004) Improving supportive and palliative care for adults with cancer: Nice Guide lines (CSG4), National Institute for Health and Care Excellence, London, UK.

4. Coleman R, Body JJ, Aapro M, Hadji P, Herrstedt J, et al. (2014) Bone health in cancer patients: ESMO clinical practice guidelines Ann Oncol 3: 124-137.

5. American Society of Clinical Oncology (2008) ASCO Annual Meeting Proceedings (Post-Meeting Edition). J Clinical Oncol 26: 20694.

6. Senkus E, Kyriakides S, Ohno S, Penault-Llorca F, Poortmans P, et al. (2015) Primary breast cancer: ESMO Clinical practice guidelines for diagnosis, treatment and follow-up. Ann Oncol 26: v8-v30.

7. Cardoso F, Costa A, Norton L, Senkus E, Aapro M, et al. (2014) ESO-ESMO 2nd international consensos guidelines for advanced breast cancer (ABC2) Breast 23: 489-502.

8. Ferris FD, Bruera E, Cherny N, Cummings C, Currow D, et al. (2009) Palliative cancer care a decade later: Accomplishment the need, next steps - from the American Society of Clinical Oncology. J Clin Oncol 27: 3052-3058.

9. Smith TJ, Temin S, Alesi ER, Abernethy AP, Balboni TA, et al. (2012) American Society of Clinical Oncology provisional clinical opinion: The integration of palliative care into standard oncology care. J Clin Oncol 30: 880-887. 
10. Pestalozzi BC (2009) Brain metastases and subtypes of breast cancer Ann Oncol 20: 803-805.

11. McCahill L, Ferrel B (2002) Palliative surgery for cancer pain. West J Med 176: 107-110.

12. Krouse RS (2009) Future directions of palliative surgery. Progress in Palliative Care 17: 261.
13. Popescu RA, Schäfer R, Califano R, Eckert R, Coleman R, et al. (2014) The current and future role of the medical oncologist in the professional care for cancer patients: A position paper by the European Society for Medical Oncology (ESMO). Ann Oncol 25: 9-15. 